EDUCATION

Research, Innovation and Solutions on-line ${ }^{(0)}$
PSYCHOLOGY

I+Dti
Electronic Journal of Research

in Educational Psychology

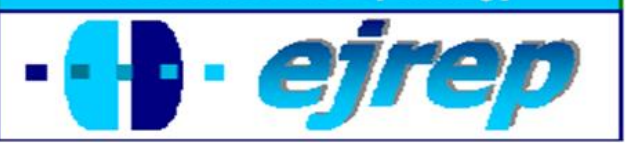

\title{
Auto-percepción de la eficacia en la escritura de alumnos sordos y oyentes de Educación Primaria y Secundaria
}

\section{Rafaela Gutiérrez Cáceres}

\author{
${ }^{1}$ Departamento de Didáctica y Organización Escolar \\ Universidad de Almería
}

\section{España}

Correspondencia: Rafaela Gutiérrez Cáceres. Departamento de Didáctica y Organización Escolar. Universidad de Almería. Ctra. Sacramento, s/n. 04120. La Cañada de San Urbano, Almería. España. e-mail: rcaceres@ual.es

(C) Education \& Psychology I+D+i and Editorial EOS (Spain) 


\section{Resumen}

Introducción. En este artículo se muestran algunos de los resultados más relevantes de una investigación centrada en el análisis de la auto-percepción sobre su eficacia en la expresión escrita por parte de los alumnos sordos y oyentes, la cual es fundamental para el adecuado desarrollo del complejo proceso escritor y de la calidad de los textos escritos.

Método. Los sujetos investigados fueron 116 alumnos: 25 de Educación Primaria y 91 de Educación Secundaria Obligatoria; 15 sordos y 101 oyentes. El proceso de recogida de datos ha consistido en la aplicación adaptada de una escala graduada sobre la auto-percepción de la eficacia en la escritura, de 10 ítems. En cuanto al procedimiento de análisis, se ha llevado a cabo una comparación y confrontación de las habilidades motivacionales del alumnado en función del nivel instructivo así como del status "sordo/oyente".

Resultados. En el análisis de resultados se ha encontrado que, en términos generales, los estudiantes sordos y oyentes de Educación Primaria y Secundaria desarrollan de manera positiva la percepción de su propia eficacia escritora. No obstante, existen unas matizaciones significativas dadas la variabilidad en las respuestas a cada uno de los ítems de la escala, así como también la diversidad existente entre el alumnado.

Discusión y Conclusión. Ante la ausencia de este tipo de estudios es preciso insistir en la necesidad de realizar investigaciones centradas en el análisis específico de los componentes afectivos de la escritura en alumnos sordos, atendiendo a los factores individuales y contextuales.

Palabras Clave: Auto-percepción de la eficacia; Composición escrita; Educación primaria; Educación secundaria; Discapacidad auditiva. 


\title{
Self-efficacy in Written Composition among Deaf and Hearing Students in Primary and Secondary Education
}

\begin{abstract}
Introduction. This article presents some of results more relevant of a research focused on the analysis of self-efficacy in written composition by deaf and hearing students, which is essential for the complex process writer and the quality of written texts.

Method. Subjects were 116 students: 25 of primary education and 91 of secondary education; 15 deaf and 101 hearing. It has been implemented a scale adapted of self-efficacy in writing, which has a 5 point-scale of ten items. Subsequently a comparison was made of the motivational skills according to the educational grade and the status "deaf/hearing".

Results. In the analysis of results it has been found that, in general terms, deaf and hearing students of Primary and Secondary Education developed positively the perception on its efficacy in writing. However, there are that take into account the variability of the answers and the diversity among the students.

Discussion and Conclusion. In the absence of this kind of studies is necessary to insist on the need to carry out research focusing on the analysis of the affective components of the writing on deaf students and taking account of the individual and contextual factors.
\end{abstract}

Keywords: Self-efficacy; Written composition; Primary Education; Secondary Education; Deafness. 


\section{Introducción}

El tema que se aborda en el presente artículo es de gran actualidad en estos momentos dentro del territorio español. Hasta hoy en día existe un $80 \%$ de analfabetismo funcional en el colectivo de personas sordas, es decir, que terminan la educación básica pero sin alcanzar un dominio adecuado del castellano, tanto en su forma oral como, sobre todo, en su modalidad escrita (Carrillo y Domínguez, 2010; Fernández y Pertusa, 2007; Jáudenes, 2009).

Dentro de la Sociedad del Conocimiento y de la Información, y de acuerdo con el desarrollo de las políticas existentes, se pretende, como uno de los ejes de la calidad educativa, asegurar el acceso eficaz a la lectoescritura, uno de los aprendizajes claves que pone de manifiesto el desarrollo de la competencia en comunicación lingüística, siendo ésta la que posibilita la adquisición de la primera competencia básica, que es “aprender a aprender", según lo establecido en la Ley Orgánica 2/2006, de 3 de mayo, de Educación. Y es en la adquisición de las competencias básicas como se asegura el ejercicio, en condiciones de igualdad, del derecho fundamental a la educación de todo ciudadano para su plena participación en la sociedad.

Sin embargo, la accesibilidad a la información y a la comunicación en la comunidad de personas sordas está aún lejos de ser un hecho. Así pues, surge la necesidad de acercarnos al conocimiento de la situación de los alumnos sordos cuando se enfrentan al aprendizaje de la lengua escrita, con miras a actuar proporcionando apoyo, concretamente en este caso, en su apropiación personal de la escritura desde una perspectiva reflexivo-crítica. "Enseñar y aprender a escribir con sentido es un derecho inalienable de las jóvenes generaciones y una obligación o compromiso de las instituciones educativas, si realmente aspiran a fomentar, fortalecer y enriquecer la emergencia del educando en el marco de una sociedad democrática, divergente y plural" (Ramos, 2009; pág. 55).

Siguiendo la perspectiva del socio-constructivismo y del enfoque comunicativodiscursivo, la escritura no es una actividad puramente de carácter técnico, en cuanto que implica el dominio de las reglas del código escrito para transcribir correctamente palabras y frases, sino que adquiere sobre todo una dimensión cognitiva, afectiva y social en la medida en que está estrechamente relacionada con la construcción de un discurso que tiene lugar en situaciones comunicativas significativas a través del diálogo y la interacción, lo que supone desarrollar un proceso recursivo y dialéctico para resolver los problemas que se plantean. Asimismo, la composición escrita constituye una actividad en la que la reflexión se hace im- 
prescindible, siendo fundamental, por tanto, la actitud estratégica frente a esta tarea (Camps y Castelló, 2007).

La escritura es, en definitiva, un instrumento de construcción cultural que permite la elaboración del pensamiento así como también el desarrollo de la comunicación y, con ello, favorecer los procesos de aprendizaje (Camps, 2004; Graham, 2008). De ahí, la necesidad de, ante todo, motivar al alumnado a participar en múltiples y ricas actividades que le ofrezcan la posibilidad de reflexionar sobre las distintas estrategias y operaciones que intervienen en el proceso de escritura, de modo que ésta tenga siempre una intención comunicativa desde la construcción de significados compartida con otros individuos.

En el proceso de enseñanza y aprendizaje de la escritura inciden diferentes factores como son, entre otros, los factores culturales, sociales, afectivos, cognitivos, metacognitivos, discursivos y pragmáticos (Arroyo y Salvador, 2009; MacArthur, Graham y Fitzgerald, 2006). Uno de los factores determinantes del proceso de escritura se refiere, en este caso, a los componentes afectivos de la lengua escrita, dada la importancia que tiene el sistema motivacional así como también los valores asociados a la actividad de escribir presentes en cada comunidad lingüística para la consecución de los objetivos propuestos y, por supuesto, las creencias atribucionales de sentido por parte del alumnado a las tareas de escritura (Boscolo, 1997; Palincsar y Klenk, 1992).

De acuerdo con los modelos teóricos sobre la composición escrita y, concretamente, en el modelo cognitivo propuesto por Hayes (1996) se evidencia la interrelación existente entre los procesos cognitivos y metacognitivos, y los elementos motivacionales/emocionales o afectivos, que se desarrolla atendiendo a la función mediadora y de instrumentalización que el individuo realiza a través de la memoria de trabajo y la memoria a largo plazo en el marco del contexto tanto físico como social y cultural. La mayoría de las investigaciones abordan de forma integrada tanto los componentes cognitivos como los afectivos-motivacionales que influyen en el aprendizaje. Ya que, en efecto, para aprender es imprescindible "poder" hacerlo, lo cual hace referencia a las capacidades, los conocimientos, las estrategias y las destrezas que son necesarias, pero además es importante "querer" hacerlo, o sea, tener la disposición, la intención, la motivación, la persistencia suficientes y la creencia en las propias posibilidades. 
No obstante, sólo recientemente hacia la década de los '90 ha surgido el interés de los estudios hacia el análisis específico de los componentes afectivos, incidiendo en la concepción que el alumnado tiene sobre la escritura de modo que permita poner en marcha los procesos de composición contribuyendo a la función epistémica (De Caso y García, 2006; Graham, Berninger y Fan, 2007; Hidi, 2007; Klassen, 2002a; Sexton, Harris y Graham, 1998; Zimmerman y Risemberg, 1997). Según los estudios realizados, en la dimensión afectiva de la lengua escrita se puede incluir, de una parte, la motivación para escribir y la actitud que adopta el escritor frente al proceso de producción del texto escrito y, de otra, la percepción que tiene de sí mismo como escritor y la auto-percepción de eficacia en la tarea (Pajares, 2003; Walker, 2003).

Concretamente, la autoeficacia como uno de los importantes predictores del éxito académico de los estudiantes, hace referencia al conjunto de creencias que tiene cada uno de ellos sobre su capacidad para aplicar adecuadamente los conocimientos y habilidades que ya posee, así como la percepción que tiene de sus posibilidades para realizar nuevos aprendizajes (Bandura, 1997). Más allá de las discrepancias en los resultados obtenidos en varias investigaciones derivadas de los aspectos metodológicos (García y Salvador, 2006), se muestra cómo la autoeficacia es uno de los determinantes motivacionales más importantes que influye en el desarrollo de la composición escrita, tanto desde el punto de vista del producto como del proceso (De Caso, García, Díez, Robledo y Álvarez, 2010; Pajares y Valiante, 1997; Salvador, 2005).

Así, los alumnos con altas expectativas de autoeficacia, o sea, los que se sienten capaces de afrontar con éxito las tareas escritoras, son quienes manifiestan una menor preocupación, mayor persistencia en la actividad y reaccionan mejor a las frustraciones, en caso de dificultades, desarrollando así una ejecución exitosa. Mientras que la anticipación del fracaso y las dudas acerca de uno mismo pueden conducir a ansiedad y garantizar el fracaso.

Asimismo, en términos generales, se ha encontrado que los alumnos con dificultades de aprendizaje son los que perciben la escritura como una actividad frustrante y a sí mismos como incapaces de escribir bien, dados sus repetidos fracasos en esta tarea (Harris, Graham y Pressley, 1992; Salvador, 2005). De este modo, se produce un círculo vicioso: el fracaso para escribir bien provoca una falta de confianza en sí mismo con respecto a esta tarea y, a su vez, esta ausencia de confianza conduce a dificultades en la expresión escrita. Pero también se ha 
observado que los alumnos valoran en exceso su capacidad y se consideran buenos escritores, al tener una percepción distorsionada de su capacidad para escribir construyendo su autopercepción de la eficacia de forma inexacta y desde diferentes fuentes (Graham y Harris, 1989a, 1989b; Harris y Graham, 2005; Klassen, 2002b, 2004; Pajares y Johnson, 1996; Sawyer, Graham y Harris, 1992). Sin embargo, en otras investigaciones (Graham, Schwartz y MacArthur, 1993) no se han detectado diferencias entre niños con y sin dificultades en el aprendizaje en la auto-percepción de la eficacia, ni diferencias en cuanto a la edad. Otros autores (Pajares y Valiante, 1999; Shell, Colvin y Bruning, 1995) en cambio, encontraron que esta habilidad se incrementa conforme se aproxima a los niveles instructivos superiores.

\section{Objetivos}

En el ámbito de la discapacidad auditiva las investigaciones se han limitado en su mayoría al análisis de la escritura desde la perspectiva del producto. Posteriormente, hacia la década de los '90 se aproximan hacia el estudio de la construcción del texto escrito desde la perspectiva del proceso (Arfè \& Perondi, 2008; Cambra, 1993; Carrillo y Domínguez, 2010; Fabbretti, Volterra y Pontecorvo, 1998; Gutiérrez, 2005; Mayer, 2010; Teruggi, 2003; van Beijsterveldt y van Hell, 2008). Sin embargo, no existen investigaciones centradas en el análisis de las múltiples dimensiones del proceso de escritura, excluyendo en este caso los factores afectivos. Partiendo de esta situación se plantea la necesidad e importancia de realizar este estudio con el fin de determinar las habilidades motivacionales del alumnado sordo en la composición escrita. Concretamente, en este estudio se pretende indagar en profundidad acerca de la auto-percepción sobre su eficacia escritora por parte de alumnos sordos y oyentes de Educación Primaria y Secundaria, es decir, conocer y analizar la percepción y valoración que cada uno posee de sus propias capacidades o habilidades escritoras. A partir de este objetivo principal, se han planteado los siguientes interrogantes o hipótesis interrogativas:

- ¿Cómo se perciben a sí mismos los alumnos, en cuanto a la efectividad en la tarea de escribir?

- ¿Se consideran capacitados para realizar las operaciones necesarias y para tener éxito en la tarea?

- ¿Se detectan diferencias entre los alumnos, en cuanto a la auto-percepción de su eficacia en la escritura, en función de la etapa educativa y del status "sordo/oyente"? 


\section{Método}

\section{Participantes}

Los sujetos investigados no fueron seleccionados al azar, sino que la muestra se ha obtenido de forma incidental u ocasional. En total participaron 116 alumnos, de los que 25 eran de $6^{\circ}$ curso de Educación Primaria y 91 de Educación Secundaria Obligatoria. Con respecto al grupo de alumnos de Educación Primaria, la media es de 12.5 años de edad, con una desviación típica de 0.3 , teniendo en cuenta que la edad mínima es 11.2 y la máxima es 13.1. En relación con el género, predominan las chicas frente al $48 \%$ que son chicos. De entre ellos, dos con 13 años de edad e hijos de padres oyentes, poseen una discapacidad auditiva de grado severo y de tipo prelocutivo y, además, emplean habitualmente audífonos así como la lengua oral y también la lengua de signos. De otra parte, el apoyo lo reciben tanto dentro del aula ordinaria como en el aula específica de apoyo, principalmente en el área de Lengua.

En cuanto a la metodología didáctica seguida con este grupo de Educación Primaria, en el centro educativo en el que se encuentra escolarizado se desarrolla un proyecto cuyos principios básicos son:

- Educar conjuntamente al alumnado sordo y oyente (educación inclusiva) con el fin de conseguir un desarrollo integral de la persona a través de los valores de tolerancia y el respeto a la diversidad.

- Potenciar una educación bilingüe utilizando tanto la lengua castellana como la lengua de signos española para la comprensión del hecho comunicativo.

Con respecto al grupo de alumnos de Educación Secundaria Obligatoria, 22 cursan el nivel instructivo de $1^{\circ}, 16$ de $2^{\circ}, 26$ de $3^{\circ}$ y 27 de $4^{\circ}$. Con respecto a la edad del alumnado, la media es de 15.11 años, siendo respectivamente de 13.49, 14.94, 15.74 y 16.27 años y con una desviación típica general de 0.78 . En cuanto al género, existe una distribución equilibrada, con un $50.93 \%$ de chicas y un $49.07 \%$ de chicos.

En este grupo de alumnos de Educación Secundaria un total de 13 son sujetos sordos, siendo 6 de ellos los que presentan una sordera profunda, otros 4 una pérdida auditiva leve y el resto una discapacidad de grado moderado-profundo. Además, respecto al tipo de prótesis 
auditivas, 7 sujetos emplean audífonos y el resto un Implante Coclear. Excepto uno, todos los alumnos sordos tienen padres que son oyentes. En estos casos, el sistema de comunicación más utilizado en el ámbito familiar se refiere al uso conjunto de las dos lenguas: la lengua oral y la lengua de signos, siendo sólo en dos casos en que se utiliza únicamente la lengua de signos, frente a cinco que emplean exclusivamente la lengua oral.

En cuanto a la metodología didáctica seguida en el Centro Ordinario de Integración Preferente de Sordos en que se encuentra escolarizado el grupo de alumnos de Educación Secundaria, se trabaja basándose en el constructivismo social, donde generalmente las actividades de escritura parten de un tema concreto con unas pautas dadas por el docente o también versan sobre algún tema libre que despierte el interés en el estudiante y haga desarrollar y fomentar la capacidad creadora en la producción de textos escritos. Asimismo, hay que destacar que el modo de comunicación prioritario es el lenguaje oral, de forma que para favorecer una adecuada recepción y comprensión de los mensajes orales en el alumnado sordo, se cuenta con el apoyo del intérprete de Lengua de Signos Española.

\section{Diseño}

En esta investigación se ha utilizado una metodología descriptiva, a partir de la observación controlada, de gran potencialidad para el estudio de la expresión escrita, teniendo en cuenta que la evaluación no se realizó en una situación artificial, sino de forma natural como una actividad práctica más en el aula y, por tanto, dirigida no sólo a los alumnos sordos sino también a los oyentes.

\section{Procedimiento}

En el proceso de recogida de datos y en relación con el conocimiento de las características personales de cada alumno, se han confeccionado unas fichas de identificación. Mediante éstas se pedía la información psicosocial más relevante a los alumnos y, en caso de duda, se solicitaba la colaboración de los profesionales.

Para evaluar la percepción que cada alumno posee de su propia capacidad como escritor, cuya definición se expone en el apartado anterior, se ha utilizado la escala de autopercepción de la eficacia en la escritura, que ha sido extraída de Harris y Graham (1992) y 
traducida por Salvador (1997). Esta escala que posee una estimación numérica comprendida entre 5 puntuaciones o valores (1. Muy en desacuerdo; 2. En desacuerdo; 3. No lo sé; 4. De acuerdo; 5. Muy de acuerdo), ha sido aplicada de forma individual, de modo que en el caso del grupo de alumnos sordos se ha adaptado en cuanto a su estructura lingüística según sus características y necesidades (ver Cuadro 1).

La fiabilidad de esta escala adaptada se ha calculado a partir de los resultados obtenidos en este estudio utilizando el índice de consistencia interna. Ha resultado ser alta, ya que el coeficiente alfa de Cronbach, en todas las dimensiones, fue superior a 0,65 y próximo a 0,80 (valor alfa $=0,784)$. El análisis de las preguntas individualmente ha mostrado que son preguntas capaces de discriminar entre las distintas opiniones de los encuestados (desviaciones típicas altas) y medias relativamente centradas en la escala (de 1 a 5 puntos).

El proceso de recogida de datos se ha realizado de forma ordenada y sistemática siguiendo las siguientes etapas:

- Entrega de fichas de identificación a los alumnos.

- Elaboración de una redacción escrita por los alumnos.

- Realización de la escala de auto-percepción sobre la eficacia escritora.

\begin{tabular}{|l|l|l|l|l|l|}
\hline ITEMS & $\begin{array}{c}\text { Muy en } \\
\text { desacuerdo }\end{array}$ & $\begin{array}{c}\text { En } \\
\text { desacuerdo }\end{array}$ & No lo sé & De acuerdo & $\begin{array}{c}\text { Muy de } \\
\text { acuerdo }\end{array}$ \\
\hline $\begin{array}{l}\text { 1.-Cuando escribo un texto, me resulta fácil encontrar } \\
\text { ideas (cuando escribo, es fácil encontrar ideas) }\end{array}$ & & & & & \\
\hline $\begin{array}{l}\text { 2.-Cuando escribo un texto, me resulta fácil organizar } \\
\text { mis ideas (cuando escribo, es fácil organizar mis } \\
\text { ideas) }\end{array}$ & & & & & \\
\hline $\begin{array}{l}\text { 3.-Cuando el profesor nos manda escribir un texto, el } \\
\text { mío es uno de los mejores }\end{array}$ & & & & & \\
\hline $\begin{array}{l}\text { 4.-Cuando escribo un texto, me resulta fácil empezar } \\
\text { (cuando escribo, es fácil empezar) }\end{array}$ & & & & & \\
\hline $\begin{array}{l}\text { 5.-Cuando escribo un texto, me resulta fácil corregir } \\
\text { lo que tengo que mejorar }\end{array}$ & & & & & \\
\hline $\begin{array}{l}\text { 6.-Cuando escribo un texto, me resulta fácil poner mis } \\
\text { ideas en frases bien hechas (cuando escribo, es fácil } \\
\text { hacer frases bien hechas) }\end{array}$ & & & & & \\
\hline $\begin{array}{l}\text { 7.-Cuando el profesor nos manda escribir una historia, } \\
\text { la mía es una de las mejores }\end{array}$ & & & & & \\
\hline $\begin{array}{l}\text { 8.-Cuando escribo un texto, me resulta fácil escribir } \\
\text { sin parar, sin descansar }\end{array}$ & & & & & \\
\hline $\begin{array}{l}\text { 9.-Cuando el profesor nos manda escribir un resumen } \\
\text { de un libro, el mío es uno de los mejores }\end{array}$ & & & & & \\
\hline $\begin{array}{l}\text { 10.-Cuando escribo un texto, me resulta fácil corregir } \\
\text { mis errores }\end{array}$ & & & & & \\
\hline
\end{tabular}




\section{Análisis de datos}

En el análisis de los datos obtenidos se han utilizado, de una parte, estadísticos descriptivos tales como la frecuencia (puntuación global de cada alumno, del grupo de alumnos y de cada ítem), la media y la desviación típica. De otra, para probar las hipótesis sobre diferencias, se aplicó la prueba "chi cuadrado" (nivel de significación: 0.05), en cada uno de los ítems de la escala y en las variables diferenciales. Para ello, se ha utilizado el programa estadístico SPSS -versión 18- a partir del cual se ha realizado una interpretación global de los datos obtenidos y, posteriormente, una comparación interindividual y una confrontación de las habilidades motivacionales del alumnado en función de la etapa educativa así como del status "sordo/oyente". Al mismo tiempo, se ha llevado a cabo una interpretación cualitativa de los resultados con el fin de evaluarlos en profundidad y analizar la auto-percepción en la escritura.

\section{Resultados}

En el análisis de las escalas de auto-percepción de la eficacia en la escritura por parte de los grupos de Educación Primaria se han encontrado los siguientes resultados más significativos.

En relación con la puntuación global de la escala, se ha obtenido una media de 3.22 (SD: 1.16). Se trata de una puntuación general que se corresponde con una auto-percepción ligeramente positiva y que se aproxima al valor "de acuerdo", según lo establecido en la escala. No obstante, hay que tener en cuenta la variedad de las respuestas de los alumnos, como se puede comprobar observando directamente las puntuaciones medias o, bien, calculando la desviación típica (véase la Tabla 1). Así, por ejemplo, los estudiantes 5, 13, 21 y 22 son los que han dado puntuaciones con valores muy positivos que indican la existencia de una percepción buena y segura sobre sus capacidades escritoras (M: 4). En contraposición a éstos, el alumno 25 es quien ha mostrado la puntuación más baja, siendo la media de la misma de 1.8. 
Tabla 1. Media y Desviación Típica de puntuaciones por alumno

\begin{tabular}{|cc|}
\hline Alumnos & M (SD) \\
\hline $\mathbf{1}$ & $3.3(1.83)$ \\
$\mathbf{3}$ & $3.1(0.57)$ \\
$\mathbf{4}$ & $2.8(1.23)$ \\
$\mathbf{5}$ & $3.7(0.95)$ \\
$\mathbf{6}$ & $\mathbf{4}(0.82)$ \\
$\mathbf{7}$ & $3(1.41)$ \\
$\mathbf{8}$ & $2.7(1.42)$ \\
$\mathbf{9}$ & $3.2(1.03)$ \\
$\mathbf{1 0}$ & $3.6(1.26)$ \\
$\mathbf{1 1}$ & $2.9(1.37)$ \\
$\mathbf{1 2}$ & $3(1.15)$ \\
$\mathbf{1 3}$ & $2.7(1.49)$ \\
$\mathbf{1 4}$ & $\mathbf{4}(0.82)$ \\
$\mathbf{1 5}$ & $3.6(1.07)$ \\
$\mathbf{1 6}$ & $3.1(1.66)$ \\
$\mathbf{1 7}$ & $3(0.67)$ \\
$\mathbf{1 8}$ & $2.9(1.45)$ \\
$\mathbf{1 9}$ & $3(1.41)$ \\
$\mathbf{2 0}$ & $3.9(0.99)$ \\
$\mathbf{2 1}$ & $2.7(1.83)$ \\
$\mathbf{2 2}$ & $\mathbf{4}(1.15)$ \\
$\mathbf{2 3}$ & $\mathbf{4}(0.82)$ \\
$\mathbf{2 4}$ & $3.2(1.03)$ \\
$\mathbf{2 5}$ & $3.4(0.52)$ \\
& $\mathbf{1 . 8}(1.13)$ \\
\hline
\end{tabular}

De otra parte, en la interpretación de los datos obtenidos en esta investigación también hay que considerar la variabilidad en las respuestas correspondientes a cada uno de los ítems de la escala (véase la Tabla 2). En efecto, mientras que en algunos ítems se han encontrado puntuaciones que se sitúan por encima de la puntuación media, en otros ítems se alcanzan valores inferiores.

Tabla 2. Media y Desviación Típica de puntuaciones por ítem

\begin{tabular}{|cc|}
\hline Ítems & M (SD) \\
\hline Ítem 1 & $3.76(1.16)$ \\
Ítem 2 & $3.92(1.04)$ \\
Ítem 3 & $2.64(1.07)$ \\
Ítem 4 & $\mathbf{4 . 0 4}(1.13)$ \\
Ítem 5 & $2.96(1.37)$ \\
Ítem 6 & $3.52(1.26)$ \\
Ítem 7 & $2.64(0.99)$ \\
Ítem 8 & $3.00(1.32)$ \\
Ítem 9 & $\mathbf{2 . 6 0}(0.96)$ \\
Ítem 10 & $3.16(1.37)$ \\
\hline
\end{tabular}


Así, la mayoría de los alumnos han manifestado una auto-percepción positiva, con una puntuación máxima de 4.04, en cuanto que consideran que cuando escriben un texto, les resulta fácil empezar (ítem 4). Mientras que es en el ítem 9 donde destacan desfavorablemente en cuanto que están en desacuerdo con la idea de que cuando el profesor les propone escribir un resumen de un libro, el suyo es uno de los mejores. En el análisis de contraste entre el grupo de alumnos sordos y el de oyentes se puede destacar que no existen grandes diferencias respecto a las puntuaciones dadas a los ítems de la escala, siendo las medias de 2.85 y 3.26 , respectivamente (véase la Tabla 3).

Tabla 3: Media de puntuaciones por ítem, según status oyente/sordo

\begin{tabular}{|ccc|}
\hline Ítems & Sordos & Oyentes \\
\cline { 2 - 3 } & $\mathbf{M}(\mathbf{S D})$ & $\mathbf{M}(\mathbf{S D})$ \\
\hline Ítem 1 & $2.5(0.71)$ & $3.87(1.14)$ \\
Ítem 2 & $3.00(1.41)$ & $\mathbf{4 . 0 0}(1.00)$ \\
Ítem 3 & $\mathbf{1 . 5 0}(0.71)$ & $2.74(1.05)$ \\
Ítem 4 & $\mathbf{4 . 5 0}(0.71)$ & $\mathbf{4 . 0 0}(1.17)$ \\
Ítem 5 & $3.00(1.41)$ & $2.96(1.40)$ \\
Ítem 6 & $3.50(2.12)$ & $3.52(1.24)$ \\
Ítem 7 & $\mathbf{1 . 5 0}(0.71)$ & $2.74(0.96)$ \\
Ítem 8 & $3.00(1.41)$ & $3.00(1.35)$ \\
Ítem 9 & $\mathbf{1 . 5 0}(0.71)$ & $\mathbf{2 . 6 9}(0.93)$ \\
Ítem 10 & $\mathbf{4 . 5 0}(0.71)$ & $3.04(1.36)$ \\
\hline
\end{tabular}

Por un lado, es necesario señalar cómo a pesar de que tanto los alumnos sordos como los oyentes coinciden en cuanto a su valoración positiva con respecto a lo enunciado en el ítem 4 (M: 4.50 y 4.00, respectivamente) y en cuanto a su valoración negativa con respecto al ítem 9 (M: 1.50 y 2.69, respectivamente), lo cierto es que aquéllos realizan unas valoraciones más extremas en relación con sus compañeros oyentes. Otro dato interesante a mencionar es que el grupo de alumnos oyentes ha destacado positivamente no sólo en el ítem 4, sino también en el ítem 2. En este sentido, se puede afirmar cómo el alumnado oyente, en comparación con el sordo, es quien está de acuerdo con la idea de que cuando escriben un texto, les es fácil organizar sus ideas (M: 4.00). 
Por otro lado, el grupo de alumnos sordos, además de que ha destacado en cuanto a su valoración positiva en relación con lo enunciado en el ítem 4, lo ha hecho también con respecto al ítem 10 (M: 4.50). Así, y en contraste con sus compañeros oyentes, los alumnos sordos afirman estar de acuerdo con la idea de que cuando escriben les resulta fácil corregir sus errores. No obstante, hay que destacar que su propia percepción acerca de que sus textos escritos o sus historias escritas sean una de las mejores, sigue siendo muy negativa (ítems 3 y 7).

Tabla 4. Media y Desviación Típica de puntuaciones por alumno

\begin{tabular}{|c|c|c|c|c|}
\hline \multirow[t]{2}{*}{ Alumnos } & $1^{\circ} \mathrm{ESO}$ & $2^{\circ}$ ESO & $3^{\circ} \mathrm{ESO}$ & $4^{\circ} \mathrm{ESO}$ \\
\hline & $\mathrm{M}$ (SD) & $\mathrm{M}(\mathrm{SD})$ & $\mathrm{M}(\mathrm{SD})$ & $\mathrm{M}(\mathrm{SD})$ \\
\hline 1 & $4(0.94)$ & $3.5(0.85)$ & $4.3(0.48)$ & $4.4(0.70)$ \\
\hline 2 & $2.9(1.37)$ & $2.6(1.43)$ & $3.1(1.10)$ & $4.0(0.94)$ \\
\hline 3 & $2.9(1.20)$ & $2.2(1.23)$ & $3.6(0.52)$ & $3.4(0.70)$ \\
\hline 4 & $3.6(0.52)$ & $3.5(0.53)$ & $3.3(0.95)$ & $2.6(0.97)$ \\
\hline 5 & $3.6(0.97)$ & $3(1.15)$ & $3(0.00)$ & $3.2(0.79)$ \\
\hline 6 & & $3.6(0.70)$ & $3(0.82)$ & $2.6(1.34)$ \\
\hline 7 & & $3(1.15)$ & 3.7 (1.57) & $3.7(0.48)$ \\
\hline 8 & $3.5(0.97)$ & $2.7(1.34)$ & $4.3(0.48)$ & $3.6(0.70)$ \\
\hline 9 & $3.2(0.63)$ & $1.6(0.52)$ & $2.3(1.34)$ & $2.7(1.16)$ \\
\hline 10 & $3.7(0.82)$ & $3.2(0.92)$ & $3.4(0.70)$ & $2.5(0.71)$ \\
\hline 11 & $3.6(0.97)$ & & $2.2(0.79)$ & $3.4(1.43)$ \\
\hline 12 & $4.2(0.92)$ & $4.5(0.53)$ & $3.5(0.71)$ & $2.8(0.92)$ \\
\hline 13 & $2.5(1.65)$ & $1.2(0.63)$ & $4.6(0.84)$ & $4.1(0.99)$ \\
\hline 14 & & $1.6(0.52)$ & $3.7(0.48)$ & $2.1(0.74)$ \\
\hline 15 & $3.8(1.03)$ & $2.6(1.26)$ & $4(0.94)$ & $3.9(0.99)$ \\
\hline 16 & $3.3(1.57)$ & & $2.5(0.97)$ & - \\
\hline 17 & $3.9(0.74)$ & $4.5(0.53)$ & - & $3.8(0.92)$ \\
\hline 18 & $3.4(0.70)$ & $3.5(1.27)$ & $2.8(0.79)$ & $2.2(1.03)$ \\
\hline 19 & $2.7(1.34)$ & & $3.6(0.70)$ & $4.4(0.52)$ \\
\hline 20 & $3.4(1.35)$ & & $2.6(1.84)$ & $3.6(0.84)$ \\
\hline 21 & $3.3(1.06)$ & & $3(0.67)$ & $3.8(0.92)$ \\
\hline 22 & $3.1(1.20)$ & & $3.5(0.97)$ & $3.6(0.70)$ \\
\hline 23 & $3.5(1.43)$ & & $3.6(0.52)$ & $2.9(0.87)$ \\
\hline 24 & $3(1.15)$ & & $3.7(0.48)$ & $3.6(0.84)$ \\
\hline 25 & $2.7(0.82)$ & & $3(1.25)$ & $4.4(0.52)$ \\
\hline 26 & & & $2.6(0.84)$ & $2.9(0.74)$ \\
\hline 27 & & & $3.9(0.57)$ & $1.6(0.52)$ \\
\hline 28 & & & & $3.4(0.84)$ \\
\hline
\end{tabular}


En referencia al grupo de Educación Secundaria se ha encontrado que la media de la puntuación global de la escala de auto-percepción de la eficacia en la escritura es de 3.32 (SD: 0.83). Se trata de una puntuación general que se corresponde con una auto-percepción ligeramente positiva cercana al valor 4 de la escala.

Contrastando los distintos grupos según el nivel instructivo de Educación Secundaria y utilizando la prueba "chi cuadrado" no se han detectado diferencias estadísticamente significativas en la percepción sobre su propia eficacia escritora (véase la Tabla 4). Partiendo de estos datos y además considerando que se trata de cuatro grupos de alumnos diversos en cuanto a características individuales y contextuales, se puede interpretar que el nivel instructivo en Educación Secundaria no es el único factor que interviene en el análisis de los resultados obtenidos.

No obstante, hay que tener en cuenta la variedad de las respuestas de los alumnos, como se puede comprobar observando directamente las puntuaciones medias o, bien, calculando la desviación típica (véase la Tabla 4). Así, por ejemplo, se ha encontrado que el sujeto 13 perteneciente al grupo de $2^{\circ}$ de ESO ha dado respuestas con unos valores muy inferiores que tienden a indicar la existencia de una percepción negativa sobre sus propias capacidades escritoras (M: 1.2). En contraposición a éste, el estudiante 13 de $3^{\circ}$ de ESO es quien ha mostrado la puntuación más alta, siendo la media de la misma de 4.6.

Tabla 5. Media y Desviación Típica de puntuaciones por ítem según nivel instructivo

\begin{tabular}{|ccccc|}
\hline Ítems & $\mathbf{1}^{\mathbf{0}}$ ESO & $\mathbf{2}^{\mathbf{0}}$ ESO & $\mathbf{3}^{\mathbf{0}}$ ESO & $\mathbf{4}^{\mathbf{0}}$ ESO \\
\cline { 2 - 5 } & $\mathbf{M}$ (SD) & $\mathbf{M}$ (SD) & $\mathbf{M}(\mathbf{S D})$ & $\mathbf{M}(\mathbf{S D})$ \\
\hline $\mathbf{1}$ & $3.86(1.32)$ & $3.25(1.34)$ & $3.69(1.01)$ & $3.52(1.34)$ \\
$\mathbf{2}$ & $3.64(0.66)$ & $2.81(1.22)$ & $3.61(0.94)$ & $3.70(1.10)$ \\
$\mathbf{3}$ & $2.86(1.04)$ & $\mathbf{2 . 5 0}(1.41)$ & $2.88(1.24)$ & $2.89(1.01)$ \\
$\mathbf{4}$ & $\mathbf{3 . 9 5}(1.25)$ & $3.37(1.41)$ & $3.81(0.89)$ & $3.18(1.21)$ \\
$\mathbf{5}$ & $3.27(1.08)$ & $3.25(1.00)$ & $3.38(0.80)$ & $3.52(0.93)$ \\
$\mathbf{6}$ & $3.73(1.03)$ & $2.81(1.28)$ & $3.31(0.97)$ & $3.33(1.07)$ \\
$\mathbf{7}$ & $2.95(0.95)$ & $\mathbf{2 . 5 0}(1.37)$ & $2.85(1.12)$ & $2.89(1.05)$ \\
$\mathbf{8}$ & $3.09(1.34)$ & $3.25(1.57)$ & $3.42(1.24)$ & $3.37(1.21)$ \\
$\mathbf{9}$ & $3.00(1.02)$ & $2.56(1.36)$ & $3.31(1.05)$ & $3.18(0.96)$ \\
$\mathbf{1 0}$ & $3.18(1.05)$ & $2.94(1.00)$ & $3.23(0.91)$ & $3.44(0.97)$ \\
\hline
\end{tabular}


De otra parte, en la interpretación de los datos obtenidos en esta investigación también hay que tener en cuenta la diversidad encontrada en las distintas respuestas a cada uno de los ítems de la escala (véase la Tabla 5). Así, se ha observado que la puntuación media más valorada se encuentra en el ítem 4 por parte de la mayoría de los estudiantes de $1^{\circ}$ de ESO, quienes han manifestado una auto-percepción positiva, con una puntuación máxima de 3.95, en cuanto que consideran que cuando escriben un texto, les resulta fácil empezar. Mientras que los ítems 3 y 7 son aquéllos cuyas puntuaciones medias son inferiores, sobre todo, en el grupo de $2^{\circ}$ de ESO, con una media de 2.50, quienes afirman que cuando el profesor les propone escribir un texto o una historia, los suyos no son los mejores, percibiéndose inferiores, lo cual podría dificultar el aprendizaje y la mejora de la composición escrita.

Tabla 6: Media y Desviación Típica por ítems en sordos y oyentes

\begin{tabular}{|c|c|c|c|c|c|c|c|c|}
\hline \multirow[t]{3}{*}{ Ítems } & \multicolumn{4}{|c|}{ SORDOS } & \multicolumn{4}{|c|}{ OYENTES } \\
\hline & $1 \mathrm{ESO}$ & 2 ESO & 3 ESO & 4 ESO & 1 ESO & 2 ESO & 3 ESO & 4 ESO \\
\hline & M (SD) & M (SD) & M (SD) & M (SD) & M (SD) & M (SD) & M (SD) & M (SD) \\
\hline \multirow[t]{2}{*}{1} & 3.60 & 4.50 & 3.00 & 3.33 & $3.94(1.39)$ & 3.07 & $3.78(1.00)$ & $3.52(1.31)$ \\
\hline & (1.14) & $(0.71)$ & (1.00) & (2.08) & & (1.33) & & \\
\hline \multirow[t]{2}{*}{2} & 3.40 & 3.50 & 3.67 & 3.67 & $3.70(0.68)$ & 2.71 & $3.61(0.99)$ & 3.65 (1.07) \\
\hline & $(0.55)$ & $(0.71)$ & $(0.58)$ & $(1.53)$ & & (1.27) & & \\
\hline \multirow[t]{2}{*}{3} & 3.00 & 3.50 & 2.33 & 3.67 & $2.82(1.07)$ & 2.36 & $2.96(1.22)$ & $2.78(1.00)$ \\
\hline & (1.00) & (2.12) & (1.53) & $(1.15)$ & & (1.34) & & \\
\hline \multirow[t]{2}{*}{4} & 3.60 & 4.50 & 3.33 & 3.67 & $4.06(1.14)$ & 3.21 & $3.87(0.92)$ & $3.09(1.20)$ \\
\hline & (1.67) & $(0.71)$ & $(0.58)$ & (1.53) & & $(1.42)$ & & \\
\hline \multirow[t]{2}{*}{5} & 4.20 & 5.00 & 3.33 & 3.00 & $3.00(0.87)$ & 3.00 & $3.39(0.84)$ & $3.61(0.94)$ \\
\hline & (1.30) & $(0.00)$ & $(0.58)$ & (1.00) & & $(0.78)$ & & \\
\hline \multirow[t]{2}{*}{6} & 4.20 & 3.50 & 2.67 & 2.00 & $3.59(1.06)$ & 2.71 & $3.39(0.99)$ & $3.48(0.99)$ \\
\hline & $(0.84)$ & $(2.12)$ & $(0.58)$ & $(1.00)$ & & $(1.20)$ & & \\
\hline \multirow[t]{2}{*}{7} & 2.80 & 3.50 & 1.67 & 3.00 & $3.00(0.93)$ & 2.36 & $3.04(0.98)$ & $2.87(1.14)$ \\
\hline & (1.09) & $(0.71)$ & (1.15) & $(0.00)$ & & (1.39) & & \\
\hline \multirow[t]{2}{*}{8} & 3.00 & 4.50 & 2.33 & 3.33 & $3.12(1.32)$ & 3.07 & $3.74(1.10)$ & $3.56(1.20)$ \\
\hline & (1.58) & $(0.71)$ & (1.15) & (1.15) & & (1.59) & & \\
\hline \multirow[t]{2}{*}{9} & 2.60 & 3.50 & 3.00 & 4.00 & $3.12(0.78)$ & 2.43 & $3.35(1.07)$ & $3.09(0.95)$ \\
\hline & (1.67) & (2.12) & (1.00) & (1.00) & & (1.28) & & \\
\hline \multirow[t]{2}{*}{10} & 3.60 & 4.00 & 2.33 & 3.00 & $3.06(1.03)$ & 2.78 & $3.35(0.88)$ & $3.56(0.94)$ \\
\hline & (1.14) & $(0.00)$ & $(0.58)$ & (1.00) & & $(0.97)$ & & \\
\hline
\end{tabular}


En el análisis de contraste entre el grupo de alumnos sordos y el de oyentes, independientemente del nivel instructivo, se puede destacar que no existen grandes diferencias en las medias de las puntuaciones a los ítems, siendo éstas de 3.36 y 3.22, respectivamente (Véase la Tabla 6). Hay que señalar que en $3^{\circ}$ sí existen diferencias, siendo las puntuaciones medias de los alumnos sordos inferiores a las de los oyentes (M: 2.77 y 3.45). Asimismo, hay que destacar que en $2^{\circ}$ de ESO también existen diferencias siendo el grupo de alumnos sordos superior al de oyentes en cuanto a auto-percepción sobre su propia eficacia escritora (M: 4 y 2.77, respectivamente).

Como se puede observar en la tabla 6, el grupo de alumnos sordos de $3^{\circ}$ de ESO es el único que posee una apreciación muy negativa sobre su propia producción escrita y concretamente en relación con lo enunciado en el ítem 7 (M: 1.67). En el caso opuesto, es el grupo de $2^{\circ}$ de ESO quienes destacan muy positivamente sobre todo en cuanto al ítem 5 (M: 5.00). Mientras que en el grupo de alumnos oyentes se ha observado que es el ítem 4 el más valorado positivamente, sobre todo, por parte del grupo de $1^{\circ}$ de ESO (M: 4.06). En cambio, es el ítem 7 el menos valorado con una puntuación media de 2.36 , encontrado en el grupo de $2^{\circ}$ de ESO.

Por último, con respecto al análisis diferencial y aplicando la prueba "chi cuadrado", en términos generales no se han detectado diferencias estadísticamente significativas entre los distintos grupos según la etapa educativa y el status "sordo/oyente" de los sujetos. Sólo existen diferencias significativas, de una parte, entre el grupo de alumnos de Educación Primaria y el de Secundaria en relación con lo enunciado en el ítem $2\left(X^{2}=9.189 ; p=0.027 ; M: 3.92\right.$ y 3.44, respectivamente) y, de otra, entre el grupo de alumnos sordos y el de oyentes en relación con el contenido del ítem $5\left(\mathrm{X}^{2}=11.788 ; \mathrm{p}=0.019 ; \mathrm{M}: 3.7\right.$ y 3.19, respectivamente).

\section{Discusión y Conclusiones}

Tras el análisis e interpretación de los resultados obtenidos en relación con la autopercepción sobre su propia eficacia escritora, se puede concluir, en términos generales, que la mayoría de los alumnos sordos y oyentes de Educación Primaria y Secundaria han desarrollado las habilidades motivacionales de forma positiva, teniendo en cuenta que la puntuación media es de 3.27, que se aproxima al valor "de acuerdo" según lo establecido en la escala aplicada. En este sentido, se puede afirmar que tanto los alumnos sordos como los oyentes, en 
general, poseen unas reales expectativas de autoeficacia, es decir, tienen unas creencias firmes sobre sus propias capacidades escritoras percibiendo la escritura como una actividad importante, de tal modo que les permiten sentirse capaces de poder afrontar dicha tarea con éxito.

También es interesante destacar cómo en esta investigación, al igual que en la realizada por Graham, Schwartz y MacArthur (1993), no se han encontrado grandes diferencias estadísticamente significativas en la percepción sobre su eficacia escritora atendiendo a la etapa educativa, siendo las medias de 3.22 en $6^{\circ}$ de Primaria, 3.28 en $1^{\circ}$ de ESO, 2.92 en $2^{\circ}$ de ESO, 3.32 en $3^{\circ}$ de ESO y 3.30 en $4^{\circ}$ de ESO. Asimismo, tampoco existen grandes diferencias entre el grupo de alumnos sordos y el de oyentes tanto en Educación Primaria como Secundaria, siendo las medias de 3.10 y 3.24 , respectivamente.

De ahí, se puede deducir que la línea de trabajo y las experiencias educativas que se están realizando en los centros educativos, están obteniendo unos logros favorables en el desarrollo de las competencias escritoras que deben conseguir en estas etapas educativas tanto los alumnos sordos como los oyentes. No obstante, hay que destacar que es necesario incidir en esas propuestas didácticas el trabajo tanto con los alumnos sordos como con los alumnos oyentes favoreciendo en la medida de lo posible su motivación hacia la realización de las actividades relacionadas con la composición escrita. Así como también se puede señalar la importancia de desarrollar una auto-percepción positiva creando un clima socio-afectivo de forma que se generen y se asimilen mensajes reales acordes con sus propias capacidades y competencias en cuanto a su propia percepción sobre sus propios textos escritos.

De otra parte, es preciso señalar que la mayoría de los alumnos, sobre todo, de Educación Primaria han destacado favorablemente en el ítem 4, siendo la puntuación media de 3.67 (cuando escriben un texto, les resulta fácil empezar). Al respecto es importante resaltar un dato significativo: el exceso de valoración con respecto a lo enunciado en el ítem 4 por parte de los alumnos sordos de Educación Primaria y de $2^{\circ}$ de ESO. Según los resultados de otros estudios realizados, esta sobrevaloración de sus habilidades escritoras se produce debido a una percepción distorsionada de su capacidad para escribir (Graham y Harris, 1989a, 1989b; Harris \& Graham, 2005; Klassen, 2002a; Sawyer, Graham \& Harris, 1992). Sería necesario, pues, indagar las razones que fundamentan estos juicios valorativos y establecer estrategias para ayudarles a conseguir una auto-percepción más realista. 
De igual modo, con respecto al contenido del ítem 5 donde se evidencian diferencias estadísticamente significativas entre el grupo de alumnos sordos y el de oyentes, esto se podría interpretar por el exceso de valoración positiva realizada por parte de aquéllos cuando afirman que les resulta fácil corregir un texto escrito. Igualmente, en relación con lo enunciado en el ítem 2 donde se manifiestan diferencias estadísticamente significativas en función de la etapa educativa, las cuales se podrían interpretar por una percepción desmesurada por parte del grupo de alumnos de Educación Primaria acerca de la idea de que les resulta fácil organizar sus ideas al escribir.

De otra parte, hay que destacar que los alumnos, en general, se perciben inferiores en cuanto que piensan que sus textos escritos no son los mejores. Así el grupo de Educación Primaria está en desacuerdo con la idea de que cuando el profesor les propone escribir un resumen de un libro, el suyo es uno de los mejores (ítem 9; M: 2.60). Este resultado coincide con uno de los hallazgos encontrados en la investigación realizada por Salvador (2005) con sujetos de Educación Primaria. De la misma forma, sucede con el grupo de Educación Secundaria, quienes afirman que cuando el profesor les propone escribir un texto o una historia, los suyos no son los mejores (ítems 3 y 7; M: 2.78 y 2.80). Esta auto-percepción negativa podría dificultar el aprendizaje y la mejora de la composición escrita.

No obstante, es necesario señalar que existen diferencias entre los alumnos sordos de Educación Primaria y los alumnos sordos de Educación Secundaria en cuanto a la valoración general de los ítems 9 y 3 , siendo éstos los que están de acuerdo con la idea de que cuando el profesor les propone escribir un resumen de un libro o un texto, el suyo es uno de los mejores (M: 1.50 y 3.27 -ítem 9-; M: 1.50 y 3.12 -ítem 3-, respectivamente). Atendiendo a estos datos, se puede deducir la incidencia del contexto escolar en la dimensión afectiva de la expresión escrita o la sobrevaloración realizada por parte del alumnado sordo de Educación Secundaria en estos ítems.

En cualquier caso, del conjunto de los datos analizados en este estudio hay que tener en cuenta la variedad de las respuestas de cada uno de los alumnos así como también la variabilidad en las respuestas correspondientes a cada uno de los ítems de la escala. Además, es preciso resaltar algunas limitaciones inherentes en este trabajo de investigación tanto en cuanto a la muestra participante como en cuanto al procedimiento metodológico de investigación. Por ello, hay que tener en cuenta que estas conclusiones no se pueden generalizar a toda la 
población, ya que inciden tanto los factores individuales como los contextuales produciéndose así diversos resultados. Si bien este estudio puede ser un punto de partida para la reflexión y la acción didáctica y, especialmente, para seguir indagando en pos de una mejora y aprendizaje de la escritura en el alumnado sordo, algunas de cuyas sugerencias más relevantes para futuras investigaciones son:

- Contrastar los resultados de esta investigación con otros estudios, realizados con otros instrumentos de obtención y análisis de datos: hablar en voz alta mientras escribe, enfoque ecológico,...

- Establecer relación entre la auto-percepción de la eficacia en la escritura y los diversos procesos cognitivos implicados: planificación, transcripción y revisión.

- Analizar la conexión entre la habilidad cognitivo-afectiva y el conocimiento y/o uso de la lengua por parte de las personas sordas (lengua oral/lengua de signos).

\section{Agradecimientos}

La importancia de esta investigación ha sido avalada por una financiación concedida por el Ministerio de Educación, mediante el Programa Nacional de Movilidad de Recursos Humanos del Plan Nacional de I-D+i 2008-2011, para la realización de una Estancia de Movilidad en el Extranjero "José Castillejo" que se ha desarrollado en la Facoltà di Scienze della Formazione de la Università Milano-Bicocca (Milán, Italia).

\section{Referencias}

Arfè, B. y Perondi, I. (2008). Deaf and hearing students'referential strategies in writing: What referential cohesion tells us about deaf students' literacy development. First Language, $28,355-374$.

Arroyo, R. y Salvador, F. (2009). Research on cognitive, social and cultural processes of written Communications. Cognitive Processing, 10, 263-268.

Bandura, A. (1997). Self-efficacy: the exercise of control. New York: Freeman and Company.

Boscolo, P. (1997). Motivazione e scrittura. Scuola Elle, 2-3, 4-8.

Cambra, C. (1993). Proceso de composición de textos narrativos escritos por alumnos sordos. Revista de Logopedia, Foniatría y Audiologia, 13, 73-78. 
Camps, A. (2004). Miradas diversas a la enseñanza y aprendizaje de la composición escrita. Lectura y Vida, 4, 14-23.

Camps, A. y Castelló, M. (2007). Las estrategias de enseñanza-aprendizaje en la escritura. In C. Monereo \& I. Solé (Coords.), El asesoramiento psicopedagógico: una perspectiva profesional y constructivista. Madrid: Alianza Editorial, 321-342.

Carrillo, M. y Domínguez, A. B. (Coords.) (2010). Dislexia y sordera: líneas actuales en el estudio de la lengua escrita y sus dificultades. Archidona: Aljibe.

De Caso, A. Ma \& García, J. N. (2006). Relación entre la motivación y la escritura. Revista Latinoamericana de Psicología, 38, 477-492.

De Caso, A. Mª García, J. N., Díez, C., Robledo, P. y Álvarez, Mª L. (2010). Mejorar las creencias de auto-eficacia para la escritura de los alumnos con dificultades de aprendizaje mejora también sus procesos y productos de escritura. Electronic Journal of Research in Educational Psychology, 8, 195-206.

Fabbretti, D., Volterra, V. \& Pontecorvo, C. (1998). Written Language Abilities in Deaf Italians. Journal of Deaf Studies and Deaf Education, 3, 231-244.

Fernández, M. P. y Pertusa, E. (2007). Estado del arte sobre la enseñanza de la lengua escrita en el niño sordo. Recuperado el 15 de mayo de 2010, de http://www.deal-leonardoeu/.

García, A. y Salvador, F. (2006). La autopercepción de eficacia en la escritura: una revisión de investigaciones. Enseñanza, 24, 79-99.

Graham, S. y Harris, K. R. (1989a). Components analysis of cognitive strategy instruction: effects on learning disabled students'compositions and self-efficacy. Journal of Educational Psychology, 81, 353-361.

Graham, S. y Harris, K. R. (1989b). Improving learning disabled students'skills at composing essays: self-instructional strategy training. Exceptional Children, 56, 201-214.

Graham, S. (2008). Research on writing development, practice, instruction, and assessment. Reading and Writing, 21, 1-2.

Graham, S., Berninger, V. y Fan, W. (2007). The structural relationship between writing attitude and writing achievement in first and third grade students. Contemporary Educational Psychology, 32, 516-536. 
Graham, S., Schwartz, S. y MacArthur, C. (1993). Learning disabled and normally achieving students'knowledge of writing and the composing process, attitude toward writing, and self-efficacy for students with and without learning disabilities, Journal of Learning Disabilities, 26, 237-249.

Gutiérrez, R. (2005). La expresión escrita de alumnos sordos. In F. Salvador (Coord.), La expresión escrita de alumnos con necesidades educativas especiales (pp. 71-87). Archidona: Aljibe.

Harris, K. R. y Graham, S. (2005). Writing better: effective strategies for teaching students with learning difficulties. Baltimore: Paul H. Brookes Publishing.

Harris, K. R., Graham, S. y Pressley, M. (1992). Cognitive-behavioral approaches in reading and written language: developing self-regulated learners. In N. N. Singh y I. L. Beale (Eds.), Learning disabilities: nature, theory and treatment (pp. 415-451). Nueva York: Springer-Verlag, 415-451.

Hayes, J. (1996). A new framework for understanding cognition and affect in writing. In C. M. Levy \& S. Ransdell (Eds.), The science of writing: theories, methods, individual differences, and applications (pp. 1-27). New Jersey: Lawrence Erlbaum Associates.

Hidi, S. (2007). Writing and motivation. Emerald Group Publishing.

Jáudenes, C. (Dir.) (2009). FIAPAS: Estudios sobre población con sordera en España. FIAPAS, 130, Monográfico.

Klassen, R. M. (2002a). Writing in early adolescence: a review of the role of self-efficacy beliefs. Educational Psychology Review, 14, 173-203.

Klassen, R. M. (2002b). A question of calibration: a review of the self-efficacy beliefs of student with learning disabilities. Learning Disability Quarterly, 25, 88-102.

Klassen, R. M. (2004). Optimism and realism: a review of self-efficacy from a cross-cultural perspective. International Journal of Psychology, 39, 205-230.

Ley Orgánica 2/2006, de 3 de mayo, de Educación. BOE, núm. 106.

MacArthur, C. A., Graham, S. y Fitzgerald, J. (eds.) (2006). Handbook of writing research. New York: Guilford Press. 
Mayer, C. (2010). The demands of writing and the deaf writer. In M. Marshark \& P. E. Spencer (Eds.), The Oxford Handbook of Deaf Studies, Language, and Education (Vol. 2). New York: Oxford University Press, 144-155.

Pajares, F. (2003). Self-efficacy beliefs, motivation and achievement in writing: a review of the literature. Reading and Writing Quarterly, 19, 139-158.

Pajares, F. (2003). Self-efficacy beliefs, motivation and achievement in writing: a review of the literature. Reading and Writing Quarterly: Overcoming Learning Difficulties, 19, 139-158.

Pajares, F. y Johnson, M. J. (1996). Self-efficacy beliefs and the writing performance of entering high school students. Psychology Schools, 33, 163-175.

Pajares, F. y Valiante, G. (1997). Influence of self-efficacy on elementary students 'writing. The Journal of Educational Research, 90, 353-360.

Pajares, F. y Valiante, G. (1999). Grade level and gender differences in the writing selfbeliefs of middle school students. Contemporary Educational Psychologist, 24, 390405 .

Palincsar, A. S. y Klenk, L. (1992). Fostering literacy learning in supporting contexts. Journal of Educational Research, 77, 683-692.

Ramos, J. (2009). Enseñar a escribir con sentido. Aula de Innovación Educativa, 185, 55-63.

Salvador, F. (1997). Dificultades en el aprendizaje de la expresión escrita. Archidona: Aljibe.

Salvador, F. (2005). Auto-percepción de la eficacia en la escritura de alumnos de Educación Primaria. Enseñanza, 23, 349-366.

Sawyer, R., Graham, S. y Harris, K. R. (1992). Direct teaching, strategy instruction, and strategy instruction with explicit self-regulation: effects on learning disabled students'compositions and self-efficacy. Journal of Educational Psychology, 84, 340352.

Sexton, M., Harris, K. R. y Graham, S. (1998). Self-regulated strategy development and the writing process: effects on essay writing and attributions. Exceptional Children, 64, 295-311. 
Shell, D., Colvin, C. y Bruning, R. (1995). Self-efficacy, attributions, and outcome expectancy mechanisms in reading and writing achievement: grade-level and achievementlevel differences. Journal of Educational Psychology, 87, 386-398.

Teruggi, L. A. (2003). Il processo di concettualizzazione della lengua scritta nei bambini sordi. In L. A. Teruggi (Ed.), Una scuola, due lingue. L'esperienza di bilingüismo della scuola dell’Infanzia ed Elementare di Cossato. Milano: Franco Angeli, 313-333.

Van Beijsterveldt, L.M. \& van Hell, J.G. (2008). Evaluative expression in deaf children's written narratives. International Journal of Language and Communication Disorders, First Article, 1-18.

Walker, B. J. (2003). The cultivation of student self-efficacy in reading and writing. Reading and Writing Quarterly: Overcoming Learning Difficulties, 19, 173-187.

Zimmerman, B. J. y Risemberg, R. (1997). Research for the future: becoming a self-regulated writer: a social cognitive perspective. Contemporary Educational Psychology, 22, 73 101. 